Research Article

\title{
An English Reading and Learning System Based on Web
}

\author{
Xiaoying He $\mathbb{1}$ \\ Xuchang University, Xuchang 461000, China \\ Correspondence should be addressed to Xiaoying He; 12007026@xcu.edu.cn
}

Received 10 August 2021; Revised 1 September 2021; Accepted 11 September 2021; Published 9 October 2021

Academic Editor: Muhammad Usman

Copyright (c) 2021 Xiaoying He. This is an open access article distributed under the Creative Commons Attribution License, which permits unrestricted use, distribution, and reproduction in any medium, provided the original work is properly cited.

Learning management system (LMS) is a web-based system used to develop, implement, and assess a specific learning process. When designing an administration learning system, it is important to understand the general architecture and roles of each level of the system. In this study, we propose a web-based English reading learning system to address the drawbacks of existing methods in English learning resource management that include login impact, system response time, and user satisfaction. English reading materials are integrated using a feature model, and the adaptive recommendation system is built using the ID3 method. The design of the proposed English reading learning system was intended to employ a hybrid of software and hardware that will benefit a wide variety of groups, especially universities, colleges, and organizations. The statistic results showed that the proposed model is capable of meeting the functional objectives of English learning resource management, as well as having a rapid and accurate response time with high user satisfaction.

\section{Introduction}

Globalization has become an essential trend of social growth in today's economic society. Different nations' economic exchanges are also required for different countries to carry out social development, in which language, as the basis of communication, plays an important role. If someone knows only one language in their own country, they will be unable to stand out in the fast-growing international society, go overseas, and help the economy grow [1]. To reduce dependency on speculating, it is important to improve learners' capacity to grasp information from the ground up by expanding their understanding of English. English is the most widely spoken and understood language in the world. The value of English learning cannot be overstated. The primary problem in front of English learning is how to manage digital education and teaching materials, network courses, and virtual learning communities in order to adapt to changes in education and teaching settings brought about by the growth of modern educational technology [2]. English reading, as a vital connection to improving the English application level [3], must be prioritized. As a result, relevant academics have investigated certain English reading techniques, systems, and platforms with the hope of improving
English reading ability using current means and technology [4].

Several researchers have proposed different teaching systems, that is, [5] suggests a revised design process for an English teaching system based on multimedia technology. The proposed system includes a stabler framework, use case diagram, and administrator interface to complete the hardware design. Lastly, the system software design is finished by enhancing the database using the error handling procedure and the background admin workflow. It was found that by using a multimedia-based English teaching system, the number of online courses could be increased, as well as the fluency of instructional videos. Similarly, a design technique for English video learning software based on smart mobile terminals is proposed in [6]. From the design goals, functional requirements, and software development environment, it briefly introduces the program. When it comes to software, it is important to understand how it works, and the precise functions of each module are described in depth. Testing shows that this approach has the benefit of a user-friendly interface, according to the results. Moreover, [7] develops a deep learning-based intelligent teaching system. The solution is divided into two components: online personalized learning recommendations and 
offline classroom quality two-way evaluation. Performance prediction and online learning behavior analysis based on deep learning are created in the online system, and learning emotion categorization is accomplished using image processing technology. It is possible to extract online learning behavior features, offline performance prediction, learning law analysis, and personalized learning recommendations using offline systems that use training target detection models, face detection models, and face segmentation models in combination with online systems. This data may also be used to evaluate and provide feedback on college teaching quality and student learning habits. According to the experimental results, the system has an easy and fast way to get information but also reduces a lot of time expenses, meets the new learning and teaching technique of integrating online and offline, and improves instructors' teaching efficiency and students' learning efficiency.

Although the approaches described above have yielded positive study findings and played an important role in enhancing English learning, there are certain issues in English learning resource management, that is, login effect, system response time, user satisfaction, on that need to be addressed further. Therefore, in this article, we propose a web-based English reading learning system. The major contributions of the paper are as follows to improve the English reading learning system:

(i) Finding word count and reading duration

(ii) Reading speed by highlighting and clicking on the desired text

(iii) Mod of complexity: if a certain book is very tough or a bit challenging, an adequate degree of difficulty level is a little easy or very easy

(iv) An appropriate vocabulary-leveled reading selection

(v) Students' practice time can be automatically recorded

The rest of the paper is organized as follows. In Section 2, a proposed system model design of the English reading and learning system. The establishment of system software design collaborative optimization model process analysis is conducted in Section 3. The experimental results and discussion are further summarized in Section 4. Finally, Section 5 concludes the paper with a summary and future research directions [8].

\section{Design of English Reading and Learning System}

2.1. System Overall Architecture Design. Internet applications in the web mode have decentralized, real-time, and non-real-time interactivity. Web can meet the individual needs of most users and provide users with more opportunities to participate in Internet content production. The process of users participating in the production of Internet content is a process of real-time interaction between users and web servers. At the same time, the information transmission between different users of the same website in the
WEB environment reflects non-real-time interaction. The "one-to-many" and "many-to-many" dissemination modes of the web have broken the information monopoly of traditional portal websites and realized mutual discussion and communication among multiple users.

According to the web model, the general structure of the English reading learning system is built on. Building the English reading learning system is meant to enable decisionmakers to create learning resource suggestion methods through effective data gathering [9] so that weak learning linkages may be targeted. Its general structure is depicted in Figure 1. A total of five layers make up the system's general architecture, including the basic layer, data layer, service layer, and application layer, as well as the user level. The basic layer is the bottom layer of the system architecture. It is mainly based on the web to support the network services of the English reading learning system to meet the system's network service requirements. The data layer is the second layer of the system framework. This layer mainly operates and manages system data, including resource data, management data, behavior data, and evaluation data. The resource data include English reading resources, cognitive style test resources, and English reading ability test scale resources. Management data includes student personal information and software information. Behavioral data include learners' behavioral data, self-designed learning plans, and semester tests. Evaluation data include answer exercise result data.

The data layer is mainly responsible for the collection, processing, exchange, and storage of the above data. The service layer is the middle layer of the overall system architecture, which is used to connect the data layer and the application layer. The service layer is mainly used to support the implementation of the system in the application layer to provide users with application services, mainly using the ASP.NET WebAPI framework, which can quickly provide APIs for HTTP clients to create web services [10]. The application layer presents the adaptive learning services that the English reading learning system ultimately provides for learners, which mainly embodies the functions of login and registration, learning resource recommendation, independent exercises, context acquisition, and learning records provided by the system. Among them, log-in and registration are used for identity verification, reading ability, and cognitive style testing; learning resource recommendation is mainly used to recommend learning resources for learners.

Autonomous practice is mainly used for learners to independently select learning modules and set learning plans according to their own learning needs. Context acquisition is mainly to obtain the environmental volume of the current user's geographical location, to judge the impact of the noise of the geographical location volume on learning. Learning records mainly record the basic information and learning behavior of current learners. The user layer is mainly the layer where the system directly contacts users and is the outermost layer of the system architecture, providing learning services for users of the English reading learning system. 


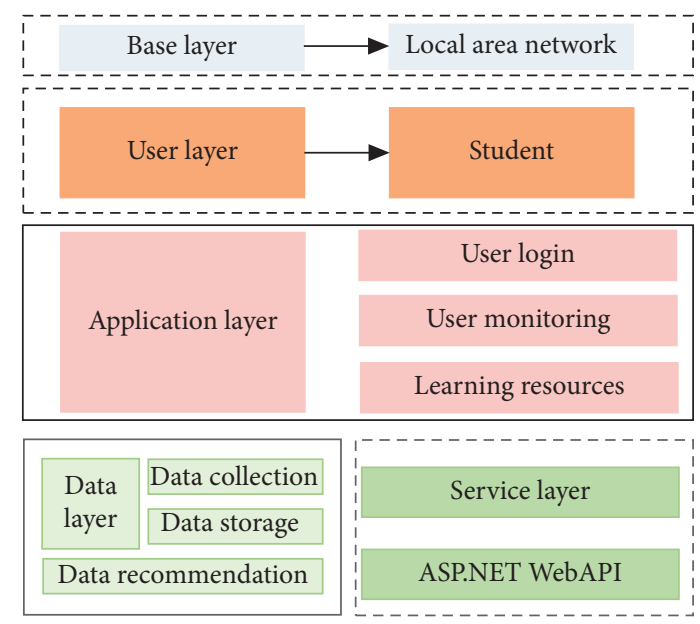

Figure 1: Schematic diagram of the overall system architecture.

2.2. Subfunction Modules. Based on the overall structure of the English reading learning system and the functions of each level, a specific analysis of the subfunction modules of the system hardware is carried out.

2.2.1. Reading Teaching Module. Teachers may use the reading teaching module to release course content, update the test question bank, and set up English reading assignments. Students can use the module to participate in reading exercises, complete unit exams, and take the final test at the end of the course. There are three primary components to the reading teaching module: reading learning, teaching business, and reading exams (or quizzes). In particular, the reading submodule is separated into two categories: time-limited reading exercises and unrestricted reading activities using a database; academic outcomes from both modes will be stored in a single location. The academic results of the two modes will be recorded in the database. The reading test submodule can be further divided into unit tests and final tests. Both tests can include objective questions and subjective questions.

2.2.2. Content Management Module. The content management module is mainly responsible for the organization, publication, modification, and deletion of articles in the system. It uses visual editing technology to provide users with a friendly operation interface and allows users to publish content in various formats such as slides, Flash animation, video, and audio [11]. Users can evaluate the usefulness of articles, and each evaluation will bring users reward points. Users of different levels will be given different permissions. Ordinary users can view and rate the content of articles, users with higher levels can publish, modify, and delete their own articles, and administrator users can manage all articles. After users share content, there will be corresponding points rewards.

2.2.3. System Administrator Module. There are a lot of things that the system administrator module is in charge of, such as setting up and maintaining basic parameters of system functioning. Semester setting and course setting comprise course administration. The semester setting determines the time interval for students to learn online, and students can only conduct online learning assignments within the defined period. In User Management, the administrator may edit all user information, such as passwords and e-mail addresses using an interface. Users and classes can be imported in batches when semester settings have been completed. These contain student ID (the unique identifier in the system), name, and gender and class/teacher information. Additionally, it allows users to upload documents in Excel format as well as replace existing entries.

2.2.4. User Management Module. After starting the system, the user directly enters the login interface. If the user does not register, user needs to register first. After successful registration, user returns to the login interface to login to the system. The specific login registration process is shown in Figure 2.

On the homepage of the software, the user chooses to enter the user management system, and then the system will automatically jump to the login module of the software. After the user enters the account and password in the login module, click "login." At this time, the entire system will perform background data in contrast. If there is no user information, it will prompt that the user does not exist. If it exists and the username and password are consistent with the background, then login to the homepage. The registration interface contains multiple edit text boxes, such as username, mobile phone, and confirm password. There are two buttons at the bottom: one is the registration button and the other is the reset button. If the user is not registered, click "register now" to jump to the registration interface. At this time, the set on click listener is mainly triggered, and then the register function is called to execute. Click "reset" to clear everything in the text box so that users can fill in personal information. It mainly uses the submit button to submit the newly filled data to the backend server by calling the commit function. The customer service system is very important whether it is in the current system or any user system. Whether the customer service system in a system is perfect is directly related to whether the design of the system is friendly or not, and whether the system design is carried out from the user's point of view. This article designs the online customer service query process, and the result is shown in Figure 3.

From Figure 3, the online customer service query module design process first, if a user has questions, he or she should go to the online customer support system and contact customer care. It will then be determined whether there is online customer service. As soon as free customer service becomes available, the user will wait in line and be shown how many others are waiting. The user will be assigned the idle customer service until there is one available in the system. After the consultation, the online customer service process is completed.

\section{System Software Design}

Based on the hardware design results of the English reading learning system, in order to further improve the application 


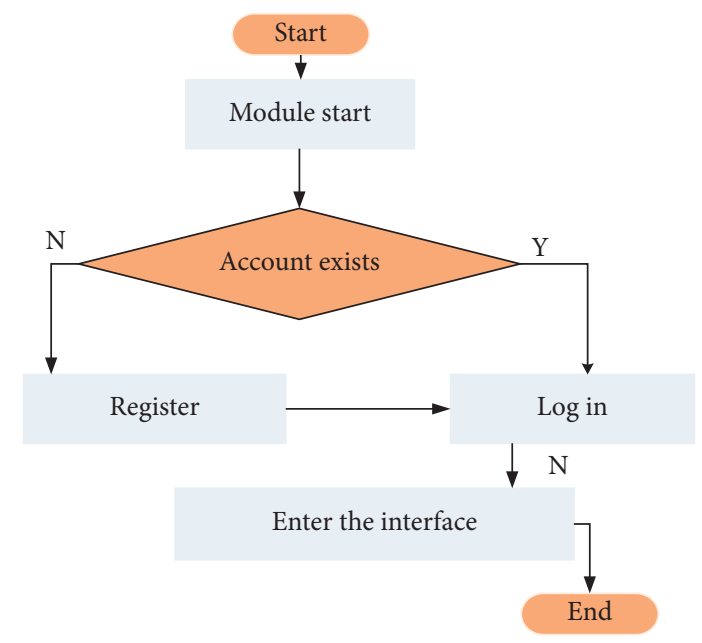

FIgURE 2: User login flowchart.

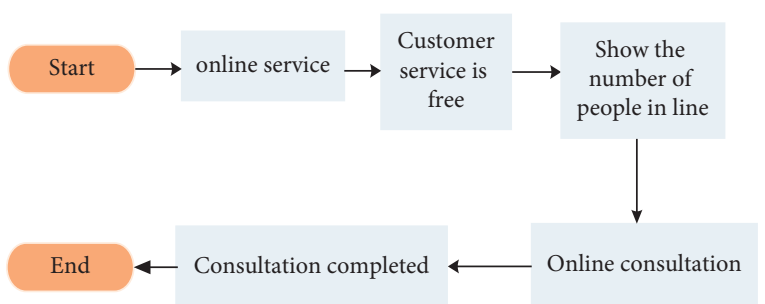

Figure 3: Schematic diagram of the workflow of the customer service query module.

performance of the system, the software algorithm is designed.

3.1. The Integration Method of English Reading Resources Based on the Feature Model. The creation of a feature model is required for the system to be able to represent the learners' learning characteristics in a timely, effective, and accurate manner. Given learners' online learning experience and system efficiency, characteristic model parameters are updated online in a timely way for learners to add friends, add collections, and do other activities that are less expensive for the system as a whole. If you are adding courseware labels, or performing other actions that require a substantial amount of processing, you should use the offline update technique. It is important to update the learner's feature model while doing so. In this case, raise the weight of the new interest subject if it is possible to retrieve it and delete the feature subject if the retrieval result is null.

The model is adjusted according to the most recently updated time $t$ and the current time $t_{1}$. The current topic interest degree is accumulated as the latest interest degree value, and the model time is adjusted to $t_{2}$. The model update calculation method is

$$
y_{i j}=\operatorname{sgn}(y)_{a} \sqrt{|y|^{a}-\left(\frac{\delta}{\left|t_{1}-t_{2}\right|}\right) \vartheta^{a}} .
$$

In the formula, $y_{i j}$ represents the interest degree after the learning courseware is updated; $|y|^{a}$ represents the learner's interest in the learning courseware calculated by the current calculation; $\vartheta^{a}$ represents the interest degree of the learning courseware recorded last time; $a$ represents the adjustment factor. The larger the value, the learner characteristic model also changes over time. The decay speed is slow and vice versa, which indicates that the decay speed is fast with time.

The feature model contains the learner's attribute information gender, age, and so on, vectorizes the information, assigns weights, and uses the cosine similarity method to calculate the background similarity between learners:

$$
S\left(p_{1}, p_{2}\right)=\frac{1}{n-m} \sum_{i=1}^{m} z_{i}-\sqrt{\left(x-\eta^{i}\right)^{T}\left(x-\eta^{j}\right)},
$$

where $S\left(p_{1}, p_{2}\right)$ represents the similarity between learners $p_{1}$ and $p_{2}, \eta^{i}$ represents the learner's $i$ th vectorized information, and $\eta^{j}$ represents the weight of the $j$ th information.

In the calculation of domain ontology similarity [12], the specific topic that the user is interested in has a corresponding weight value, and the similarity is calculated by combining the domain ontology and its learner's scoring information on the learning courseware. The user-interested topic dataset is $E=\left\{e_{1}, e_{2}, \ldots, e_{N}\right\}, \varphi\left(e_{c}, e_{v}\right)$ is the two topics of interest $e_{c}$ and $e_{v}$, which are the closest to the same category node in the ontology, and the semantic similarity calculation formula of the topics of interest $e_{c}$ and $e_{v}$ in the ontology is as follows:

$$
S\left(e_{c}, e_{v}\right)=\frac{\varpi\left(e_{c}, e_{v}\right)}{\mu\left(e_{c}\right)+\mu\left(e_{v}\right)} \times d_{k},
$$

where $₫\left(e_{c}, e_{v}\right)$ represents the semantic similarity of interest topics $e_{c}$ and $e_{v}, \mu\left(e_{c}\right)$ and $\mu\left(e_{v}\right)$ both represent the depth of the path from the root node of the same resource in the ontology to the user label, and $d_{k}$ represents the path length from the root node of the same resource in the ontology to the nearest common ancestor nodes of $e_{c}$ and $e_{v}$. The data range of $S\left(e_{c}, e_{v}\right)$ is $[0,1]$, when $e_{c}=e_{v}$; that is, the two topics are the same, $S\left(e_{c}, e_{v}\right)=1$; the similarity value of the interest topic increases as the depth of the common ancestor node increases.

In the integration of English reading resources [13], the integrated data resources include learner scoring dataset, predicted scoring value, and data recommendation. Set the learning courseware dataset as $Y=\left\{y_{1}, y_{2}, \ldots, y_{n}\right\}$, the learner dataset as $X=\left\{x_{1}, x_{2}, \ldots, x_{n}\right\}$, and the learner's scoring of the courseware denoted as $\left\{\left(y_{1}, h_{1}\right),\left(y_{2}, h_{2}\right), \ldots,\left(y_{n}, h_{n}\right)\right\}$; the grading set of $y_{k}$ is $\left\{\left(z_{1}, h_{1}\right),\left(z_{2}, h_{2}\right), \ldots,\left(x_{n}, h_{n}\right)\right\}$, and the set of courseware that $X_{k}$ is interested in is $X_{q}=\left\{y_{n}, \ldots, y_{m}\right\}$. To predict the scoring data of $X_{k}$ courseware on $A=\left\{x_{i}, \ldots, x_{j}\right\}$, the specific equation is

$$
T_{i j}=\left\{\frac{\left[\sum_{i, j=1}^{n}\left(x_{i}-x_{j}\right)^{2}\right]}{\left[\sum_{n=1}^{N}\left(x_{n} \times \sigma_{k}\right)^{2}\right]}\right\}^{2} .
$$


In (4), $T_{i j}$ represents the scoring result; $\sigma_{k}$ represents the similarity of the scores of learners. It was determined that the similarity of interest themes as well as semantics and scores could be merged into the integration of English reading materials, using the analysis, and calculations described above.

\subsection{Adaptive Recommendation Algorithm for English Reading} Resources Based on ID3 Algorithm. Aiming at the fusion of learner characteristics and resource model characteristic data obtained in the context of English reading learning, an adaptive recommendation algorithm for English reading based on the ID3 algorithm is designed. From the basic idea of the algorithm, the initial recommendation of resources and the adaptive recommendation of resources based on the ID3 algorithm, the design of the adaptive recommendation algorithm for English reading is described [14].

The algorithm selects the attribute with the highest information gain as the test attribute of the current node, which minimizes the amount of information required for data classification in the result division and reflects the minimum randomness of the division. For the known classification attributes, the decision tree is constructed from top to bottom, divide, and conquer. Starting from the root node, the data sample set is calculated, and the data sample is divided into several subsample sets according to the calculation results, and each subsample set forms a new subnode. When the given conditions are not satisfied, the process of iterating to establish the decision tree continuously. In the generated decision tree, each non-leaf node corresponds to a non-category attribute, the branch represents the value of the attribute, and the path from the tree root to the leaf node corresponds to a rule, so as to obtain the expression rules and realize the recommendation of resources. ID3 algorithm is suitable for processing large-scale data, with strong learning ability, simple algorithm principle, easy understanding, fast classification speed, and easy to explain classification rules.

Taking $V$ as the training set, the target attribute $G$ of $V$ has $M$ possible class label values, $G=\left\{g_{1}, g_{2}, \ldots, g_{m}\right\}$; the probability of $G_{i}$ appearing in all samples is $I$, $I=1,2, \ldots, M$; then the information entropy contained in the training set $V$ is

$$
\zeta(V)=\sum_{i=1}^{n} \varphi_{i} \log _{2} \kappa_{v}
$$

where $\varphi_{i}$ represents the probability that any sample belongs to $G_{i}$. Since the information is encoded in binary, the logarithm in the formula is based on 2. Assuming that attribute $R$ has $f$ different values, thus dividing into $f$ sample subsets $V=\left\{v_{1}, v_{2}, \ldots, v_{f}\right\}$, the information entropy of the sample subsets after dividing $V$ by attribute $R$ is

$$
\zeta_{R}(V)=\frac{\int_{i}^{n} f\left|V_{f}-v_{f}\right|}{\left|V_{i}\right|},
$$

where $\left|V_{i}\right|$ represents the number of samples contained in sample subset $V_{i}$ and $\left|V_{f}-v_{f}\right|$ represents the number of valid samples contained in sample set $V$. Assuming that the sample dataset before the division is $V_{1}$, and the attribute $B$ is used to divide the sample set $V_{1}$; the information gain $\partial\left(V_{1}\right)$ of dividing $V_{1}$ according to the attribute $B$ is the entropy of the sample set $V_{1}$ minus the entropy of the sample subset after the attribute $B$ is divided $V_{1}$ :

$$
\partial\left(V_{1}\right)=\Phi\left(V_{1}\right)-\Lambda\left(V_{1}\right) .
$$

Calculate the information gain of each attribute according to formulae (5)-(7). The greater the information gain, the purer the sample subset of attributes and the more conducive to classification. Select the attribute with the largest information gain as the test attribute of the sample set, construct a node, create a branch for each value of the attribute, and divide it continuously so as to build a decision tree and obtain rules, so as to recommend English reading and learning resources for learners in subsequent learning. In order to further improve the effect of resource recommendation, the multiobjective optimization problem of online learning resources is decomposed into many single objective subproblems by Chebyshev decomposition method, and a unique weight vector is given to correspond to it.

(1) Calculate the weight vector of online learning resources. To solve the online learning resource recommendation model based on multiobjective optimization strategy $[15,16]$, first decompose the online learning resource problem so that each online learning resource subproblem corresponds to a $\theta$ vector and calculate the weight vector of the online learning resource subproblem:

$$
\theta_{i j}^{2}=\frac{\widehat{u} \cdot\left|\theta_{\max }-\theta_{\min }\right|}{\operatorname{rand}(N, 1)} .
$$

In the formula, $\widehat{u}$ represents the subproblem in the multiobjective optimization problem, $\theta_{\max }$ represents the number of online learning resources, and $\theta_{\min }$ represents the amount of resource redundancy [17].

(2) Vector sorting of online learning resources subproblems. Because the neighborhood strategy in the evolutionary algorithm is to use similar vectors to optimize the current vector, it is necessary to calculate the distance between each weight vector and sort according to the distance to find the neighborhood of the current particle. The distance between each weight vector can be calculated by the following:

$$
D\left(p_{i}, p_{j}\right)=A_{j i}(L)-A_{j}(L) .
$$

(3) Determine the current learning resource neighborhood. According to (9), the particle neighborhood is obtained, that is, several learning resources that are similar to the current learning resources. Let $O$ be the number of learning resources randomly selected among the number of learning resources, and $O=2$. 
TABLE 1: Verification of front-end login function.

\begin{tabular}{|c|c|}
\hline Test & Specific description \\
\hline Test type & Management interface \\
\hline Test mode & Manual \\
\hline Test target & Registered account users can login to the system \\
\hline Prespecification & After registering an account on the website, the background system passes the review \\
\hline Expected result & $\begin{array}{c}\text { After login, the user can communicate in the download area and the discussion and exchange area; if the login fails, a } \\
\text { prompt message will be displayed }\end{array}$ \\
\hline Testing process & Enter the username and password in the login page and click login \\
\hline Test results & Pass \\
\hline
\end{tabular}

(4) Solve the average fitness of the learning resource domain. If two learning resources $s_{i}(x)$ and $s_{i}(y)$ are randomly selected from the neighborhood, the average value of the neighborhood is

$$
\overline{S_{i}}=\frac{1}{N} \sum_{i=1}^{n}\left(\overline{s_{i}(x)}-\overline{s_{i}(y)}\right)^{2} .
$$

(5) Give particles the ability to explore new areas. In the later stage of optimization, the algorithm is easy to fall into local optimization, and it is difficult to find the global optimal solution. Therefore, the ability to explore new regions is added to the algorithm used in the online learning resource recommendation method, in order to increase the ability $\Psi$ of the algorithm to explore other regions in the solution space, improve the convergence ability of the algorithm, and explore new regions:

$$
\Psi=\frac{\left|R_{s}\right|}{\overline{S_{i}} \times p_{s}} .
$$

\section{System Test}

As part of a simulation experiment, a test is conducted to determine whether or not the planned English reading and learning system based on the Web is successful and complete. Based on comparing and contrasting English video learning software based on intelligent mobile terminals and enhanced English teaching systems using multimedia technology, it is possible to determine the advantages of the developed system.

4.1. System Function Test. The experiment tests the foreground login and background resource management of the designed English reading learning system, which are described in Tables 1 and 2, respectively.

In Tables 1 and 2, we can see that the front-end login and backend resource management functions of the developed system passed the tests. This shows how effective and capable the system in this article is at managing the teaching of the English reading learning system is.

4.2. System Performance Test. System performance verification is carried out to further evaluate the application impact of the developed system in real applications.
Different techniques are tested based on the system response time and user satisfaction. The results are shown in Figure 4. Figure 4 shows that as the number of online users continues to grow, the system response time has continued to increase. As a result, there has been an increasing trend in design methods for English teaching systems based on multimedia technology and English video learning software based on smart mobile devices. Through the comparison of specific data, when the number of online users is 300 , the response time of the designed system is $0.3 \mathrm{~s}$, the response time of the improved design method of English teaching system based on multimedia technology is $1.2 \mathrm{~s}$, and the response time of the design method of English video learning software based on intelligent mobile terminal is $0.9 \mathrm{~s}$. When the number of online users is 600 , the response time of the designed system is $0.9 \mathrm{~s}$, the response time of the improved design method of English teaching system based on multimedia technology is $1.7 \mathrm{~s}$, and the response time of the design method of English video learning software based on intelligent mobile terminal is $1.5 \mathrm{~s}$. The comparison shows that the response time of the designed system is shorter, indicating that it is running speed is faster and the system performance is better.

On the other hand, a comparison of user satisfaction with the system is made. The survey subjects are 200 students from different classes at a university. To teach online English reading to the case class, a variety of systems is employed. The questionnaire will be handed to the students at the end of the course. The questionnaire has a 40-minute response period to determine the students' level of satisfaction with the educational environment. Table 3 summarizes the results.

Table 3 shows that the student's satisfaction with the developed system is considerably greater than the enhanced design technique of the English teaching system based on multimedia technology and the design method of English video learning software based on an intelligent mobile terminal, as shown in Table 3. There is a substantial difference between this system's maximum score of 95 and the greatest score of older systems of 79. Thus, the proposed system is superior to the old system not only in terms of system function but also in terms of performance.

4.3. Evaluation of Collective Learning Behaviors. Reading behaviors were split into two categories as part of the proposed web-based English Reading Learning System: learning and learning-unrelated behaviors. Individual, intergroup, and intragroup reading were among the learning- 
TABLE 2: Verification of background resource management functions.

\begin{tabular}{|c|c|}
\hline Test & Specific description \\
\hline Test type & Management interface \\
\hline Test mode & Manual \\
\hline Test target & The administrator implements editing processing on resources \\
\hline Prespecification & Backstage administrator login \\
\hline Expected result & $\begin{array}{c}\text { The client analyzes whether the resource has been edited before or is empty and then submits the } \\
\text { relevant resource to the database }\end{array}$ \\
\hline Testing process & $\begin{array}{l}\text { In the embedded development introduction management interface, click the category-based search } \\
\text { option, select a resource point, and click the edit, delete, and add buttons }\end{array}$ \\
\hline Test results & Pass \\
\hline
\end{tabular}

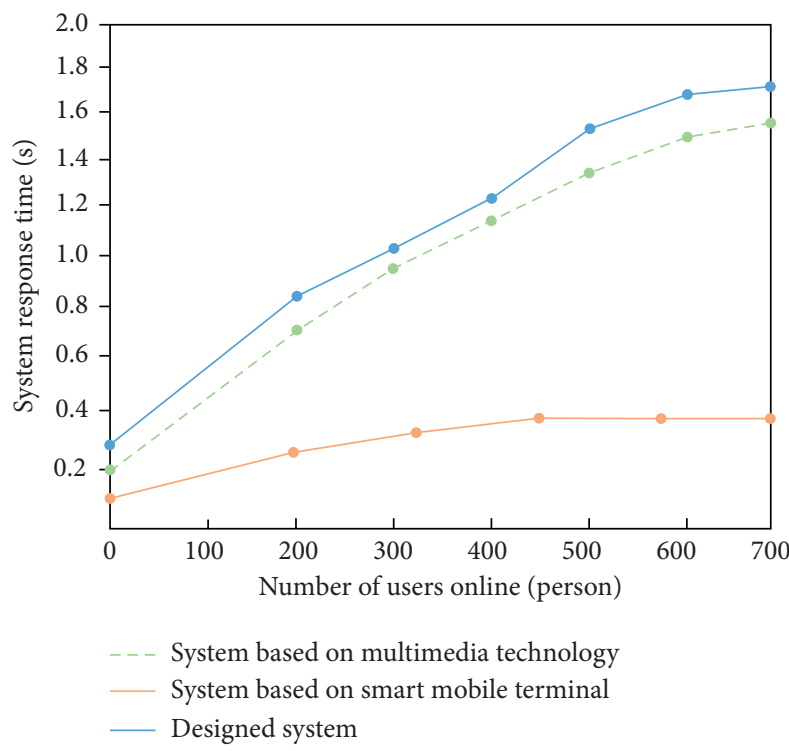

FIgURE 4: System response time comparison results.

Table 3: Comparison of user satisfaction.

\begin{tabular}{lccc}
\hline No. of students & Designed system & Multimedia technology & Mobile technology \\
\hline 20 & 87 & 75 & 70 \\
40 & 89 & 77 & 74 \\
60 & 89 & 76 & 70 \\
80 & 88 & 70 & 69 \\
100 & 87 & 72 & 75 \\
120 & 90 & 73 & 78 \\
140 & 91 & 73 & 73 \\
160 & 93 & 71 & 70 \\
180 & 95 & 76 & 75 \\
200 & 90 & 79 \\
\hline
\end{tabular}

related behaviors. The frequency of each type of behavior in each group is depicted in Figure 5. According to chi-square analysis, the frequency of learning-related and learningunrelated behaviors was significantly greater in the experimental group than in the control group $\left(\chi^{2}(1,1)=91.32, \mathrm{p}\right.$
0.05). As seen in Figure 5, students in the experimental group concentrated on reading activities. Figure 5 also shows that benefit from collaborative reading behavior both intraand intergroup, particularly intergroup behavior due to the usage of peer-to-peer internet-based telephony. 


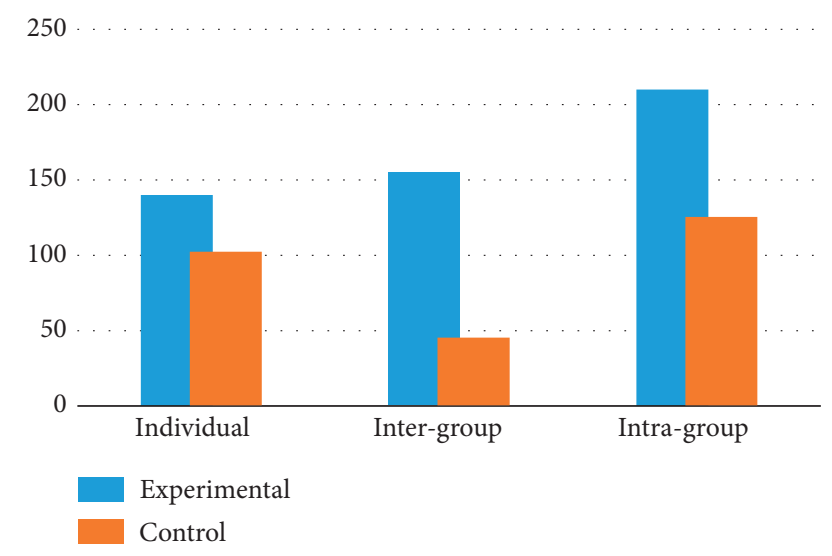

Figure 5: The analysis results of frequency of collective learning behaviors.

\section{Conclusion}

In conventional approaches, many factors contribute to the problems of inadequate English learning resource management, which delayed system response time and also low user satisfaction. One of the most significant shortcomings of English learners is their inability to accurately grasp the basic daily conversations of fluent English speakers. This article aims to create a web-based English reading learning system to implement administration functions, planned system login, and registration. To accomplish English reading resource integration and resource adaptive recommendation, the feature model and ID3 algorithm are utilized. The testing findings demonstrate that the developed system can meet the predefined functional objectives of English learning resource management and has a quick response time and high user satisfaction. The proposed model indicates that the system has a high application value and will reduce dependency on speculating. Moreover, it can improve learners' capacity to grasp information from the ground up by expanding their understanding of English. In the future study, the number of users should be expanded, and more efforts should be made to improve their learning ability by offering effective and resilient comprehensive reading and active reading techniques.

\section{Data Availability}

The data used to support the findings of this study are available from the corresponding author upon request.

\section{Conflicts of Interest}

The author declares no known conflicts of interest or personal relationships that could have appeared to influence the work reported in this paper.

\section{References}

[1] U. L. Igbokwe, E. N. Nwokenna, C. Eseadi, C. S. Ogbonna, and A. R. Ogbuagu, "Intervention for burnout among English education undergraduates: implications for curriculum innovation," Medicine, vol. 98, no. 26, p. 16219, 2019.
[2] H. Chen and J. Huang, "Research and application of the interactive English online teaching system based on the internet of things," Scientific Programming, vol. 2021, no. 1, p. 10 2021.

[3] X. Ma, "Fixed-point tracking of English reading text based on mean shift and multi-feature fusion," Journal of Intelligent and Fuzzy Systems, vol. 40, no. 1, pp. 1-11, 2020.

[4] S. Yang, "Construction of video courses of physical education and health education in colleges and universities under the MOOC platform," Mobile Information Systems, vol. 2021, Article ID 9925838, 2021.

[5] Y. Sun, "Improved design of English teaching system based on multimedia technology," Modern Electronics Technique, vol. 41, no. 10, pp. 129-132, 2018.

[6] Z. M. Liao, "Design of English video learning software based on intelligent mobile terminal," Video Engineering, vol. 42, no. 8, pp. 100-103+117, 2018.

[7] J. Y. Chen, Z. Wang, J. Y. Chen, Z. Q. Chen, and H. B. Zheng, "Design and research on intelligent teaching system based on deep learning," Computer Science, vol. 46, no. 1, pp. 550-554+576, 2019.

[8] J. Huang, "An internet of things evaluation algorithm for quality assessment of computer-based teaching," Mobile Information Systems, vol. 2021, Article ID 9919399, 2021.

[9] X. B. She, Q. H. Zhan, and C. X. Wu, "Multi-node information resource allocation recommendation algorithm based on collaborative filtering," Computer Simulation, vol. 38, no. 6, pp. 419-423, 2021.

[10] H. Wu, "Multimedia interaction-based computer-aided translation technology in applied English teaching," Mobile Information Systems, vol. 2021, Article ID 5578476, 10 pages, 2021.

[11] D. Wu and Q. Pan, "Permission and content management model based on ASP.NET technology and three-layer network architecture," Journal of Intelligent and Fuzzy Systems, vol. 39, no. 6, pp. 8857-8866, 2020.

[12] T. Kim, Y. R. Lee, B. Kang, and E. G. Im, "Binary executable file similarity calculation using function matching," The Journal of Supercomputing, vol. 75, no. 2, pp. 607-622, 2019.

[13] B. Mather and G. Yuan, "Onward and upward: distributed energy resource integration [guest editorial]," IEEE Power and Energy Magazine, vol. 18, no. 6, pp. 16-19, 2020.

[14] C. Li, W. Li, and S. B. Tsai, "Automatic classification algorithm for $m$ data association rules in wireless networks," Wireless Communications and Mobile Computing, vol. 2021, no. 7, Article ID 5591387, 9 pages, 2021.

[15] Q. Yuan, "Network education recommendation and teaching resource sharing based on improved neural network," Journal of Intelligent and Fuzzy Systems, vol. 39, no. 4, pp. 5511-5520, 2020.

[16] W. Li, "Multimedia teaching of college musical education based on deep learning," Mobile Information Systems, vol. 2021, Article ID 5545470, 10 pages, 2021.

[17] J. Cheng and H. Wang, "Adaptive algorithm recommendation and application of learning resources in english fragmented reading," Complexity, vol. 2021, Article ID 5592534, 11 pages, 2021. 